\title{
Differentiation between Acute Rejection and HCV Recurrence after Living Donor Liver Transplantation: Is there A Role for C4d?
}

\author{
Hany Dabbous*, Mohammed Sakr, ImanMontasser, ShimaaYousef, Nedal Hegazy, Kamal Mamdouh, Hany \\ Sayed, Mohamed Rady, Magda El Monayery and Mahmoud El Meteini
}

Department of Hepatology, Ain Shams University, Egypt

Submission: April 24, 2017; Published: May 30, 2017

"Corresponding author: Hany M Dabbous, Ain Shams Center for Organ Transplant, College of Medicine, Ain Shams University, Abbasia, Cairo, Egypt, Email: drhdabbous@gmail.com

Abstract

Introduction: Liver biopsy represents the gold standard for diagnosis of acute rejection and HCV recurrence after liver transplantation; nevertheless discrimination can be difficult due to similar display of alterations in liver specimen. Therefore, a specific marker expressed only in rejection but not in HCV recurrence would be a great asset to differentiate between both conditions. The aim of this study wasto assess the role of tissue C4d complement fragments in liver biopsy as a marker for differentiating between acute rejection and HCV recurrence in recipients post LDLT.

Methods: A case control study on 25 recipients after liver transplantation with the suspicion of either acute rejection or HCV disease recurrence, patients were classified according to pathological finding into two groups, Group 1: patients with acute rejection (n=13), Group 2: patients with HCV recurrence $(\mathrm{n}=12)$, The $\mathrm{C} 4 \mathrm{~d}$ was evaluated by immunohistochemical staining of the formalin-fixed, paraffin-embedded tissue in different liver compartments.

Results: C4D staining of all the studied tissue compartments (Sinusoids, portal vein endothelium , hepatic vein endothelium , arterial internal elastic lining, portal stroma, bile ducts ) had high specificity (100\% )and positive predictive value (100\%) in diagnosis of rejection cases except portal vein endothelium. (Specificity 91.7\%, positive predictive value $88.9 \%$ ).

Conclusion: Tissue C4D staining was almost present in rejection cases only; Further studies on larger cohort are required to stand on standard diagnostic criteria for $\mathrm{C} 4 \mathrm{~d}$ to be included in diagnosis of acute rejection after liver transplantation as its role in other organ transplantation

Keywords: Liver transplantation; Acute rejection; Hepatitis C recurrence; Liver biopsy

\section{Introduction}

Liver transplantation is a well established procedure for curative treatment of various liver diseases [1]. Inspite of continuously improving immunosuppressive therapy, acute graft rejection is still a complication after liver transplantation [2]. Both acute rejection and HCV recurrence often display the same clinical picture with rising serum transaminases, elevated bilirubin level and deterioration of productive liver function in the absence of perfusion deficit [3]. Liver biopsy represents the gold standard for diagnosis of both acute rejection and HCV recurrence; nevertheless discrimination can be highly difficult due to a quite similar display of alterations in liver specimen
[3]. Both conditions are characterized by predominantly portalbased inflammation, which may involve bile ducts and portal venous endothelium [4]. Correct differentiation between acute rejection and recurrent hepatitis is very important because of differences in treatment [5].

Therefore a specific marker expressed only in rejection but not in HCV recurrence cases would be a great asset to differentiate patients with clinically suspicious symptoms in order to validate rejection diagnosis [6]. The aim of this prospective study wasto assess the role of tissue $\mathrm{C} 4 \mathrm{~d}$ complement fragments in liver biopsy as a marker for differentiating between acute rejection and HCV recurrence in recipients post LDLT. 


\section{Advanced Research in Gastroenterology \& Hepatology}

\section{Patients and Methods}

This prospective, case-controlled study included 25 Egyptian patients with HCV related end stage liver disease who underwent living donor liver transplantation (LDLT) in Ain Shams Center for Organ Transplantation (ASCOT) and Egypt Air Hospital, Cairo, Egypt from January 2015 to December 2015. The study was approved by the Ethics Committee of Ain Shams University Hospitals, Cairo, Egypt in accordance with local research governance requirements. Informed written consent was obtained from each participant before enrollment in the study. The trial was registered with the federal clearinghouse for randomized trials: ClinicalTrials.gov (NCT02209675).

Criteria adopted for exclusion were patients with other etiologies for liver disease as indication for transplantation , patients with other causes of post transplant elevated liver functions as post transplant biliary stricture or vascular liver diseases (if unresolved) and patients with active CMV infection ( proved by positive CMV PCR or positive inclusion bodies by histopathology).

Liver core biopsy tissue were fixed in $10 \%$ buffered formalin and 3-4 micrometer thick sections were cut from the paraffin embedded tissue for hematoxylin and eosin (H\&E) and trichrome stains for morphological evaluation. The slides were evaluated and scored on the basis of previously described pathological criteria for acute cellular rejection and recurrent hepatitis (Macsween's pathology of the liver, 2011).

\section{C4d immunostaining}

C4d immunostaining was performed on 3 micrometer thick sections cut from formalin-fixed, paraffin-embedded sections

The C4d polyclonal antibody against C4d (CELL MARQUE) was used for immunostaining. For antigen retrieval, we used Dako Target Retrieval solution PH9.

\section{Interpretation of C4d immunostaining}

C4d deposition in the sinsuoids, endothelium of the portal veins or hepatic veins, the arteriolar internal elastic lamina, portal stroma and bile duct epithelium was documented.

The intensity of staining was recorded as follows (0) negative, $(1+)$ weak, $(2+)$ moderate and $(3+)$ strong. Regarding sinusoidal staining, the staining of more than $10 \%$ of sinusoidal compartment in the liver (intensity of $1+$ or greater) was considered as positive. Diffuse staining was defined as staining involving more than $50 \%$ of the sinusoids whereas focal staining involved less than $50 \%$ of the sinusoids [7].

Staining of the hepatocytes and staining of elastic fibers were regarded as non specific and considered negative. Re-evaluation of patients within one month of treatment to determine the clinical response to treatment by liver function tests was done.

The studied patients were classified according to pathological finding into two groups (Table 1):
Table 1: Pathological findings of the post transplant liver biopsies of the studied cases $(n=25)$.

\begin{tabular}{|c|c|c|}
\hline & $\mathbf{N}$ & $\%$ \\
\hline Portal tract inflammation & 20 & 80.0 \\
\hline Bile duct inflammation & 2 & 8.0 \\
\hline Bile duct degenerative changes/ damage & 5 & 20.0 \\
\hline Ductopenia & 1 & 4.0 \\
\hline Bile duct proliferation & 9 & 36.0 \\
\hline Interface hepatitis & 11 & 44.0 \\
\hline $\begin{array}{l}\text { Endothelitis(venous endothelial } \\
\text { inflammation) }\end{array}$ & 6 & 24.0 \\
\hline Parenchymal inflammation & 13 & 52.0 \\
\hline Parenchymal ballooning & 10 & 40.0 \\
\hline \multicolumn{3}{|l|}{ Steatosis } \\
\hline Negative & 14 & 56.0 \\
\hline Mild & 10 & 40.0 \\
\hline Severe & 1 & 4.0 \\
\hline Sinusoidal Dilatation & 1 & 4.0 \\
\hline Fibrosis & 17 & 68.0 \\
\hline \multicolumn{3}{|l|}{ Metavir Score* } \\
\hline A1F1 & 5 & 41.7 \\
\hline $\mathrm{A} 1 \mathrm{~F} 2$ & 5 & 41.7 \\
\hline A2F1 & 1 & 8.3 \\
\hline \multirow[t]{2}{*}{$\mathrm{A} 2 \mathrm{~F} 2$} & 1 & 8.3 \\
\hline & Mean \pm SD & Range \\
\hline HAI score ${ }^{* *}$ & $6.2 \pm 2.0$ & $2.0-9.0$ \\
\hline BANFF Score*** & $3.4 \pm 0.5$ & $3.0-4.0$ \\
\hline
\end{tabular}

*Metavir score and ${ }^{* *}$ Hepatitis Activity Index $(\mathrm{HAI})$ were done for $\mathrm{HCV}$ recurrent cases $(n=12)$

${ }^{* * *}$ BANFF score for acute rejection cases $(n=13)$

Group 1: patients with acute rejection $(n=13)$

Group 2: patients with HCV recurrence $(n=12)$

\section{Statistical Methods}

Statistical analyses were performed using the SPSS version 18 computer database (SPSS Inc., Chicago, IL, USA). Descriptive statistics were done for quantitative parametric data as minimum \& maximum of the range as well as mean \pm SD (standard deviation) and for numerical non parametric data as median and st $\& 3^{\text {rd }}$ inter-quartile range, while they were done for qualitative data as number and percentage.

Inferential analyses were done for quantitative variables using independent t-test in cases of two independent groups with parametric data and Mann Whitney $U$ in cases of two independent groups with non parametric data.

In qualitative data, inferential analyses for independent variables were done using Chi square test for differences between proportions as well as McNemar test for agreement between paired categorical data. ROC curve was used to evaluate 


\section{Advanced Research in Gastroenterology \& Hepatology}

the performance of different tests differentiate between certain groups.

The level of significance was taken at $\mathrm{P}$ value $<0.050$ is significant, otherwise is non-significant. Sensitivity, specificity, positive predictive value, negative predictive value and diagnostic accuracy of tissue C4d were calculated.

\section{Results}

The demographic features of the studied population showed that there were 22 men (88\%) and 3 women (12\%) with their age ranging from 19 to 63 years (mean 49.2 \pm 9.7 ). Concerning the pre-transplant Child scoring system; one patient (4\%) was child A , seven patients(28\%) were child B and seventeen patients were child C (68\%), their MELD score ranged from (7-26) mean (16.4 \pm 4.4$)$. As regard the pre-transplant clinical presentation, ascites was a common presentation in our patients (64\%) who underwent liver transplantation.

Regarding the post transplant laboratory abnormalities ,serum bilirubin and alkaline phosphatase were significantly higher in rejection group $(5.5 \mathrm{mg} / \mathrm{dl}, 245.5 \mathrm{IU} / \mathrm{L})$ respectively compared to HCV recurrence group $(1.1 \mathrm{mg} / \mathrm{dl}, 145.5 \mathrm{IU} / \mathrm{L})$ respectively. Results of the present work showed high diagnostic performance of Alkaline phosphatase, total and direct bilirubin in diagnosis of rejection, Area under the curve for total and direct bilirubin was $0.871,0.852$ respectively; ( $p$ value $0.003,0.004$ respectively) while AUC for alkaline phosphatase was 0.761 (p value 0.034 ). The histopathological findings in the examined cases are summarized in table one, BANFF score was done for acute rejection cases (mean 3.4, range 3-4).

Tissue C4D staining was present in rejection cases only (Figure 1-3), this was statistically significant in all studied tissue compartments except hepatic vein endothelium and arterial internal elastic lining (AEL) did not reach statistical significance (Figure 1); also there were agreement between histopathological features of rejection in biopsy results and tissue C4D staining (Table 2).

Table 2: Agreement between histopathological features of rejection in biopsy results and tissue C4D staining in prediction of rejection*\#.

\begin{tabular}{|c|c|c|c|c|}
\hline \multicolumn{2}{|c|}{ C4D staining } & \multicolumn{2}{|c|}{$\begin{array}{c}\text { Pathological features of } \\
\text { Rejection }\end{array}$} & \multirow{2}{*}{ \#P } \\
\cline { 3 - 4 } & Positive & Negative & \\
\hline \multirow{2}{*}{ Sinusoids } & Pos & $6(24.0 \%)$ & $0(0.0 \%)$ & \multirow{2}{*}{0.238} \\
\hline & Neg. & $7(28.0 \%)$ & $12(48.0 \%)$ & \\
\hline $\begin{array}{c}\text { Portal vein } \\
\text { endothelium }\end{array}$ & Pos & $8(32.0 \%)$ & $1(4.0 \%)$ & \\
\hline & Neg. & $5(20.0 \%)$ & $11(44.0 \%)$ & \\
\hline
\end{tabular}

\begin{tabular}{|l|c|c|c|c|}
\hline \multirow{2}{*}{$\begin{array}{l}\text { Hepatic vein } \\
\text { endothelium }\end{array}$} & Pos & $1(4.0 \%)$ & $0(0.0 \%)$ & \\
\hline & Neg. & $12(48.0 \%)$ & $12(48.0 \%)$ & \multirow{2}{*}{0.003} \\
\hline & Neg. & $9(36.0 \%)$ & $12(48.0 \%)$ & \\
\hline
\end{tabular}

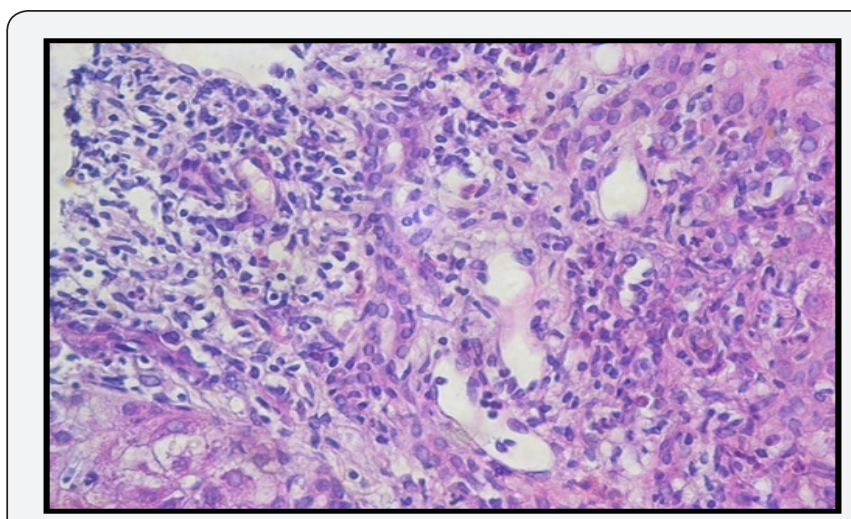

Figure 1: Liver biopsy from a patient with acute (cellular) rejection (H\&E stain $\mathrm{x} 400$ ).

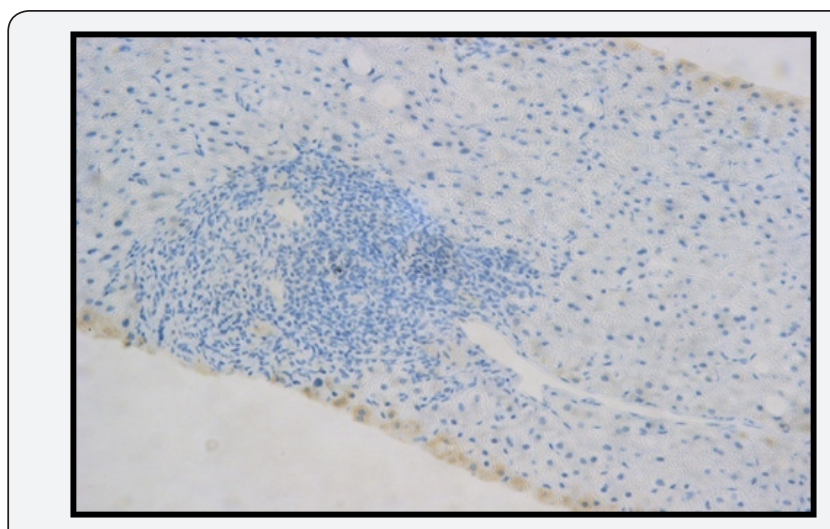

Figure 2: Liver biopsy from a patient with acute (cellular) rejection (H\&E stain $\mathrm{x} 400$ ).

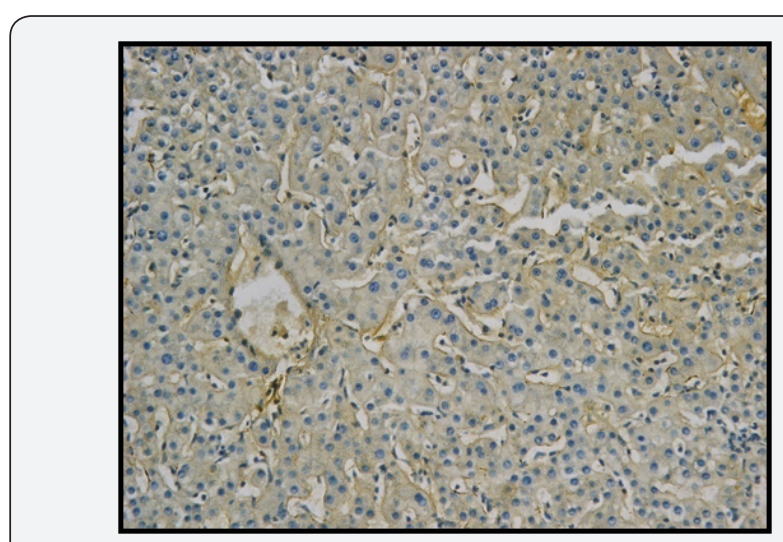

Figure 3: Sinusoidal c4d staining (IHC X200). 


\section{Advanced Research in Gastroenterology \& Hepatology}

C4D staining of all the studied tissue compartments (Sinusoids, portal vein endothelium, hepatic vein endothelium, arterial internal elastic lining (IEL) portal stroma, bile ducts had high specificity (100\%) and positive predictive value (100\%) in diagnosis of rejection except portal vein endothelium. (Specificity 91.7\%, positive predictive value 88.9\%) (Table 3).

Table 3: Diagnostic accuracy of C4D staining of the studied tissue compartments in diagnosis of rejection.

\begin{tabular}{|c|c|c|c|c|c|}
\hline Character & Sensitivity & Specificity & PPV & NPV & DA \\
\hline Sinusoids & $46.20 \%$ & $100.00 \%$ & $100.00 \%$ & $63.20 \%$ & $72.00 \%$ \\
\hline $\begin{array}{l}\text { Portal vein } \\
\text { endothelium }\end{array}$ & $61.50 \%$ & $91.70 \%$ & $88.90 \%$ & $68.80 \%$ & $76.00 \%$ \\
\hline $\begin{array}{l}\text { Hepatic vein } \\
\text { endothelium }\end{array}$ & $7.70 \%$ & $100.00 \%$ & $100.00 \%$ & $50.00 \%$ & $52.00 \%$ \\
\hline Arterial internal & $7.70 \%$ & $100.00 \%$ & $100.00 \%$ & $50.00 \%$ & $52.00 \%$ \\
\hline \multicolumn{6}{|l|}{ elastic lining(IEL) } \\
\hline Portal stain & $38.50 \%$ & $100.00 \%$ & $100.00 \%$ & $60.00 \%$ & $68.00 \%$ \\
\hline Bile ducts & $30.80 \%$ & $100.00 \%$ & $100.00 \%$ & $57.10 \%$ & $64.00 \%$ \\
\hline
\end{tabular}

*Percentages were calculated from total cases $(\mathrm{N}=25)$,

\#McNemar test

We found that the sensitivity and diagnostic accuracy of tissue C4d in sinusoids can be further increased by serial application of tissue $\mathrm{C} 4 \mathrm{~d}$ in both liver sinusoids and portal vein endothelium ( sensitivity $76.9 \%$, specificity $91.7 \%$ and diagnostic accuracy 84.0\%) (Table 4).

Table 4: Diagnostic characteristics of serial application of tissue C4D staining of Sinusoids and Portal vein endothelium in diagnosis of rejection.

\begin{tabular}{|c|c|}
\hline Character & Value \\
\hline Sensitivity & $76.9 \%$ \\
\hline Specificity & $91.7 \%$ \\
\hline Predictive positive value(PPV) & $90.9 \%$ \\
\hline Predictive negative value(PNV) & $78.6 \%$ \\
\hline Diagnostic accuracy(DA) & $84.0 \%$ \\
\hline
\end{tabular}

Regarding the management of the studied patients, all proven HCV recurrence cases $(n=12)$ received combined Sofosubivir, Daclatasivir and ribavirin adjusted between 400 and $800 \mathrm{mg} / \mathrm{d}$ according to level of hemoglobin. While patients with rejection $(n=13)$, eight patients required increasing dose Tacrolimusin addition to Mycophenolatemofetilwith oral steroid while five patients received Cyclosporine and Mycophenolatemofetil in addition to oral steroid; Everolimus was added in 3 cases.

\section{Discussion}

Liver transplantation is a well established procedure for curative treatment of various liver diseases [1]. Hepatitis $C$ is the most common indication for liver transplantation. Recurrence of HCV is universal leading to graft failure in up to $40 \%$ of all patients [2]. Controversy remains regarding the significance of $\mathrm{C} 4 \mathrm{~d}$ and its staining pattern in liver transplantation. So far, there is no consensus for the anatomic pattern of $\mathrm{C} 4 \mathrm{~d}$ staining in liver allograft rejection. The differences of staining protocols including the type of materials (frozen or formalin-fixed tissue), type of $\mathrm{C} 4 \mathrm{~d}$ antibodies, and antigen retrieval can affect the results of C4d staining [8]. The Discovery of C4d as a clinical marker for Antibody mediated rejection (AMR), in the early nineties marked a turning point in the history of solid organ transplantation [9].

They showed that patients with suspected antibodymediated injury in the renal graft had a linear C4d staining pattern in peritubular capillaries and that the presence of $\mathrm{C} 4 \mathrm{~d}$ was associated with impaired graft function Remarkably, these initial publications received relatively little attention in the transplant community. At the turn of the century the group of [10] tested for presence of C4d along with other markers of endothelial activation or injury in renal transplant biopsies suspected of AMR. A few years later, the correlation with graft survival that had been reported on in 1993 was confirmed by other groups [11,12] This led to general acceptance of the usefulness of C4d in the identification of acute AMR and in 2003 'C4d' was incorporated in the Banff classification [13]. Although a diffuse C4d staining is defined as positive, the definition and clinical significance of 'minimal' and 'focal' C4d staining remain debated issues. Most experts consider a focal staining pattern as a red flag, especially when detected on paraffin-embedded tissue or in the presence of donor-specific antibody and/or suspicious histopathological features [13]. In the current study, tissue C4D staining was almost present in rejection cases only, this was statistically significant in all studied tissue compartments except hepatic vein endothelium and arterial internal elastic lining did not reach statistical significance. There was agreement between histopathological diagnosis and tissue C4D staining of the examined tissue compartments in prediction of rejection except with Hepatic vein endothelium, arterial internal elastic lining (IEL) and bile ducts, therefore C4d can be considered a new marker to differentiate patients with rejection.

The first report concerning the role of $\mathrm{C} 4 \mathrm{~d}$ as a potential factor differentiating acute liver rejection from HCV hepatitis relapse dates back to 2005 [2], who demonstrated statistically 
significant difference in C4d expression in liver biopsies from acute graft rejection patients as compar with the control group and with HCV infection relapsers. Also, Lorho et al. [13] obtained C4d-positive reactions in 33\% of acute rejection patients, in all chronic rejection cases and in $14 \%$ patients from the control group, with no positive reactions in HCV relapsers, the results obtained from chronically rejected livers can additionally suggest the role of humoral mechanisms also in this process [7]. However on the other hand, Sakashita and his colleagues (2007) who conducted their study on 764 patients classified into two groups (crossmatch-negative patients 749) and (crossmatchpositive patients 15) and concluded that C4d deposit in ABOcompatible/identical liver transplantation, unlike in kidney or cardiac transplantation, is not always specifically associated with rejection histology; however, extensive staining of C4d suggested possible humoral rejection in cross match-positive patients [14]. Regarding C4d staining on various structures on liver tissue, the present work revealed that $\mathrm{c} 4 \mathrm{~d}$ was deposited significantly along sinusoids in six patients with histopathological features of rejection $(46.2 \%)$ ( $p=0.007)$, this could be explained as liver sinusoids parallel the capillary compartments in other organs in which the blood flow is slowed and is no longer pulsatile (with resulting pressures in the range of $2-5 \mathrm{mmHg}$ ) that these conditions may foster the interaction of anti- HLA alloantibodies with endothelial cell antigens in liver sinusoids and allow deposits of complement from the blood stream similar to C4d deposits seen in peritubular capillaries of kidney allografts with antibody mediated rejection and also there is a correlation between the presence of HLA antibodies with donor specificity in ABO-compatible liver allograft recipients and the presence of C4d deposits detected by the IF technique in the sinusoids [15].

This agrees with Watson [14] who reported that C4d deposits were found by immunoflorescence along sinusoids [16]. Also, [7] correlated this with the presence of donor-specific antibodies (DSAs). A linear/granular sinusoidal pattern of C4d immunoflorescence was noted in 18 of 28 biopsy samples obtained after transplantation from patients with positive cross-match and detectable donor-specific alloantibody (posXM/DSA) [15]. C4d staining was negative in liver sinusoidal endothelium in seven patients with histopathological features of rejection in liver biopsy this may be due to the use of immunohistochemistry in parafinized tissue that has proven to be less sensitive than immunofluorescence results and frozen liver tissues [15]. thought that the major drawback of their study was the lack of data from frozen tissue and concluded that $\mathrm{C} 4 \mathrm{~d}$ on paraffin sections tended to be less sensitive than on frozen sections. They investigated both staining techniques on interand intra-observer variation and found a favorable result for immunoflorescence stained frozen sections ( $\kappa=0.9$ (IF) versus $\kappa=0.3$ (IHC) [17]. However, in that study, C4d staining by both staining techniques was not investigated in relation to graft outcome over time. Also, in the Banff criteria it was noted that
C4d staining by immunoflorescence showed more positivity and is possibly more sensitive, but both $\mathrm{C} 4 \mathrm{~d}$ staining techniques show strong relations with detectable donor-specific alloantibodies [18].

C4d staining of the arterial internal elastic lining (IEL) was positive in only one patient (7.7\%) this agree with [7] who concluded that C4d staining of the arterial IEL is considered a nonspecific immunoflorescence event even arterial immunoflorescence staining in frozen tissue cannot be considered specific for antibody mediated rejection in the transplantation of ABO-compatible liver allograft [15]. In our study, we reported c4d deposits in portal stroma in five patients with histopathological features of rejection, this against [7] that documented no correlation was found between C4d deposits in the portal stroma and the presence of circulating donor-specific alloantibody and/ or a positive crossmatch detectable in liver transplant recipients. They interpret these non sinusoidalimmunoflorescence staining patterns as not characteristic for antibody mediated rejection [15].

Regarding C4d deposits in portal vein endothelium this study shows significant staining in eight patients $(61.5 \%)$ ( $p$ value 0.006), this agree with Schmeding et al. (2006) [2], who reported that half or more of patients diagnosed with acute rejection showed C4d deposits along portal vessels, mainly portal veins, and C4d may be a useful marker to distinguish acute rejection from recurrent hepatitis C [2]. However the results of [7] showed weak or moderate portal vein immunohistochemistry staining pattern in several biopsy samples with strongly positive sinusoidal immunoflorescence staining [15], which previous authors have reported with the immunohistochemistry technique in liver allografts $[19,20]$. That suggests that portal vein endothelium immunohistochemistry staining is not as sensitive as sinusoidal immunofluorescence findings.

In the current work, it was also crucial to determine that C4D staining of all the studied tissue compartments had high specificity $100 \%$ and positive predictive value $100 \%$ in diagnosis of rejection except portal vein endothelium (specificity 91.7\%, positive predictive value $88.9 \%$ ). This in concordance with [19] who conducted his study on 12 patients with rejection and reported the significance of $\mathrm{C} 4 \mathrm{~d}$ deposits in liver tissue as well as positive crossmatch and detectable donor-specific alloantibody in these patients [21]. Also [20] reported that C4d is deposited by immunohistochemistry mostly but not only in periportal areas [22]. The C4d localization correlated with the cellular components of the host immune response concentrated in the periportal regions of the liver allograft tissue. On the other hand, Schmeding et al. [2], performed a prospective analysis by ELISA measurement of C4d concentration in cryo-preserved liver biopsies of LTX patients who had either experienced acute rejection, hepatitis-C recurrence or displayed no pathological alterations (controls). Opposed to their immunohistologically based findings in paraffinized tissue they were unable to detect 
significant differences of C4d concentration in ELISA of cryopreserved liver tissue [2].

Most centers involved in the management of transplant recipients have incorporated routine $\mathrm{C} 4 \mathrm{~d}$ staining in diagnostic pathology evaluation of all renal allograft biopsies [23]. A solid base for regular C4d staining of biopsied allograft tissue is now established for heart transplantation and pancreas transplantation [24]. For other transplanted organs such as the lung, the usage of $\mathrm{C} 4 \mathrm{~d}$ staining is still controversial.

In liver and short bowel transplantation C4d seems to have no additional diagnostic value We found that the sensitivity and diagnostic accuracy of tissue C4d in sinusoids can be further increase by serial application of tissue $\mathrm{C} 4 \mathrm{~d}$ in both liver sinusoids and portal vein endothelium (sensitivity (76.9\%) and diagnostic accuracy $84.0 \%$ ) in order not to miss true case. This means C4d staining considered positive if sinusoids positive or even negative sinusoids with positive portal vein endothelium.

Finally, tissue C4D staining was almost present in rejection cases only; further studies on larger cohort of patients are required to stand on standard diagnostic criteria for $\mathrm{C} 4 \mathrm{~d}$ to be included in diagnosis algorithm of acute rejection after liver transplantation as its role in other organ transplantation.

\section{References}

1. Charlton M (2003) Natural history of hepatitis C and outcomes following liver transplantation. Clin Liver Dis; 7(3): 585-602.

2. Schmeding M, Dankof A, Krenn V, Krukemeyer MG, Koch M, et al. (2006) C4d in acute rejection after liver transplantation - a valuable tool in differential diagnosis to hepatitis $\mathrm{C}$ recurrence. Am J Transplant; 6(3): 523-30.

3. Regev A, Molina E, Moura R, Bejarano PA, Khaled A, et al. (2004) Reliability of histopathologic assessment for the differentiation of recurrent hepatitis $\mathrm{C}$ from acute rejection after liver transplantation. Liver Transpl 10(10): 1233-1239.

4. Petrovic LM (2006) Early recurrence of hepatitis C virus infection after liver transplantation. Liver Transpl 12(11 Suppl 2): S32-S37.

5. Jain A, Ryan C, Mohanka R, Orloff M, Abt P, et al. (2006) Characterization of CD4, CD8, CD56 positive lymphocytes and C4d deposits to distinguish acute cellular rejection from recurrent hepatitis C in postliver transplant biopsies. Clin Transplant 20(5): 624-633.

6. Schmeding M, Kienlein S, Röcken C, Neuhaus R, Neuhaus P, et al. (2010) ELISA-based detection of C4d after liver transplantation - A helpful tool fordifferential diagnosis between acute rejection and HCV-recurrence? Transplant Immunology 23(4): 156-160.

7. Kozlowski T, Andreoni K, Schmitz J, Hayashi PH, Nickeleit V, et al. ( 2012) Sinusoidal C4d deposits in liver allografts indicate an antibodymediated response: Diagnostic considerations in the evaluation of liver allografts, Liver Transplantation 18(6): 641-658.

8. Feucht HE, Schneeberger H, Hillebrand G, Burkhardt K, Weiss M, et al. (1993) Capillary deposition of C4d complement fragment and early renal graft loss. Kidney Int 43(6): 1333-1338.
9. Collins AB, Schneeberger EE, Pascual MA, Saidman SL, Williams WW, et al. (1999) Complement activation in acute humoral renal allograft rejection: diagnostic significance of $\mathrm{C} 4 \mathrm{~d}$ deposits in peritubular capillaries. J Am SocNephrol 10(10): 2208-2214.

10. Berger SP, Roos A, Daha M (2005) Complement and the kidney: what the nephrologist needs to know in 2006? Nephrol Dial Transplant 20(12): 2613-2619.

11. Herzenberg AM, Gill JS, Djurdjev 0, et al. (2002) C4d deposition in acute rejection: an independent long-term prognostic factor. J Am SocNephrol 13(1): 234-241.

12. Racusen LC, Colvin RB, Solez K, Hayashi PH, Nickeleit V ( 2003) Antibody-mediated rejection criteria-an addition to the Banff 97 classification of renal allograft rejection. Am J Transplant 3(6): 708714 .

13. Lorho R, Turlin B, Aqodad N, Triki N, de Lajarte-Thirouard AS, et al. (2006) C4d: A Marker for Hepatic Transplant Rejection. Transplant Proc 38(7): 2333-2334.

14. Watson R, Kozlowski T, Nickeleit V, Woosley JT, Schmitz JL, et al. ( 2006) Isolated donor specific all antibody mediated rejection after $\mathrm{ABO}$ compatible liver transplantation. Am J Transplant 6(12): 3022-3029.

15. Seemayer CA, Gaspert A, Nickeleit V, et al. ( 2007) C4d staining of renal allograft biopsies: a comparative analysis of different staining techniques. Nephrol Dial Transplant 22(2): 568-576.

16. Montgomery RA, Zachary AA, Racusen LC, Leffell MS, King KE, et al. (2000) Plasmapheresis and intravenous immune globulin provides effective rescue therapy for refractory humoral rejection and allows kidneys to be successfully transplanted into cross-match-positive recipients. Transplantation 70(6): 887-895.

17. Jain A, Ryan C, Mohanka R, Orloff M, Abt P, et al.(2006) Characterization of $\mathrm{CD} 4, \mathrm{CD} 8, \mathrm{CD} 56$ positive lymphocytes and C4d deposits to distinguish acute cellular rejection from recurrent hepatitis $C$ in postliver transplant biopsies. Clin 20(5): 624-633.

18. Kamar N, Lavayssie 're L, Muscari F, Selves J, Guilbeau-Frugier C, et al.( 2009) Early plasmapheresis and rituximab for acute humoral rejection after ABO-compatible liver transplantation. World J Gastroenterol 15(27): 3426-3430.

19. Bellamy CO, Herriot MM, Harrison DJ, Bathgate AJ (2007) C4d immunopositivity is uncommon in ABOcompatible liver allografts, but correlates partially with lymphocytotoxic antibody status. Histopathology 50(6): 739-749.

20. Musat AI, Agni RM, Wai PY, Pirsch JD, Lorentzen DF, et al. ( 2011) The significance of donor-specific HLA antibodies in rejection and ductopenia development in $\mathrm{ABO}$ compatible liver transplantation. Am J Transplant 11(3): 500-510.

21. Racusen LC, Colvin RB, Solez K, Mihatsch MJ, Halloran PF, et al. (2003) Antibody-mediated rejection criteria-an addition to the Banff 97 classification of renal allograft rejection. Am J Transplant 3(6): 708714.

22. Fedrigo M, Gambino A, Tona F, et al. (2010) Can C4d immunostaining on endomyocardial biopsies be considered a prognostic biomarker in heart transplant recipients? Transplantation 90(7): 791-798.

23. Glanville AR (2010) Antibody-mediated rejection in lung transplantation: myth or reality? J Heart Lung Transplant 29(4): 395400 .

24. Neil DA, Hubscher SG (2010) Current views on rejection pathology in liver transplantation.TransplInt 23(10): 971-983. 


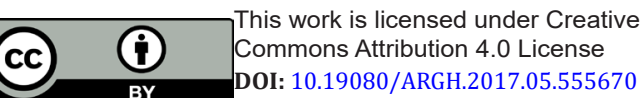

\section{Your next submission with JuniperPublishers} will reach you the below assets

- Quality Editorial service

- Swift Peer Review

- Reprints availability

- E-prints Service

- Manuscript Podcast for convenient understanding

- Global attainment for your research

- Manuscript accessibility in different formats

( Pdf, E-pub, Full Text, audio)

- Unceasing customer service

Track the below URL for one-step submission https://juniperpublishers.com/online-submission.php 